\title{
Spectral solution of ODE-IVPs by using SHBVMs
}

Cite as: AIP Conference Proceedings 2293, 100002 (2020); https://doi.org/10.1063/5.0026404

Published Online: 25 November 2020

P. Amodio, L. Brugnano, F. lavernaro, and C. Magherini
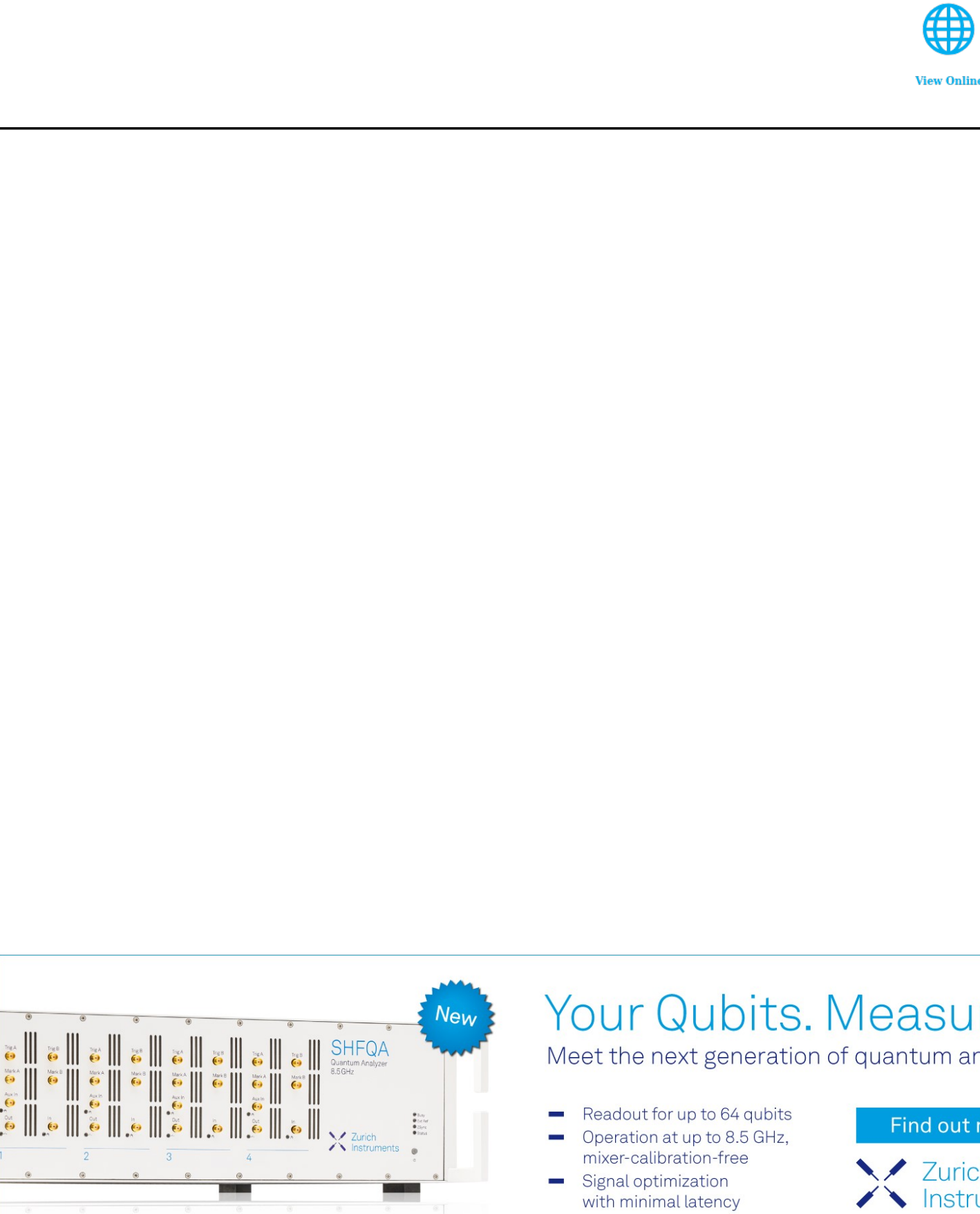

Your Qubits. Measured.

Meet the next generation of quantum analyzers

- Readout for up to 64 qubits

- Operation at up to $8.5 \mathrm{GHz}$. mixer-calibration-free

- Signal optimization with minimal latency
Find out more

Zurich

Instruments 


\title{
Spectral solution of ODE-IVPs by using SHBVMs
}

\author{
P. Amodio*, L. Brugnano ${ }^{\dagger, a}$, F. Iavernaro* and C. Magherini ${ }^{* *}$ \\ *Dipartimento di Matematica, Università di Bari, Italy. \\ ${ }^{\dagger}$ Dipartimento di Matematica e Informatica "U. Dini”, Università di Firenze, Italy. \\ ** Dipartimento di Matematica, Università di Pisa, Italy. \\ a)luigi.brugnano@unifi.it
}

\begin{abstract}
Recently, Hamiltonian Boundary Value Methods (HBVMs), have been used as spectral methods in time for effectively solving multi-frequency, highly-oscillatory and/or stiffly-oscillatory problems. A complete analysis of their use in such a fashion has been also carried out, providing a theoretical framework explaining their effectiveness. We report here a few numerical examples showing their potentialities to provide a fully accurate solver for general ODE problems.
\end{abstract}

Keywords: Spectral methods, Legendre polynomials, Hamiltonian Boundary Value Methods, SHBVMs.

PACS: $02.30 . \mathrm{Hq} ; 02.70 . \mathrm{Hm}$; 02.60.-x. MSC: 65L05; 65P10.

\section{INTRODUCTION}

Hamiltonian Boundary Value Metods (HBVMs) is a class of energy-conserving Runge-Kutta methods for efficiently solving Hamiltonian problems [6, 7] (see also the monograph [3] and the recent review paper [4]). In particular, a $\operatorname{HBVM}(k, s)$ method, $k \geq s$, is the $k$-stage Runge-Kutta method defined by the following Butcher tableau:

$$
\frac{\boldsymbol{c} \mid \mathscr{I}_{s} \mathscr{P}_{s}^{\top} \Omega}{\mid \boldsymbol{b}^{\top}}, \quad \mathscr{I}_{s}=\left(\int_{0}^{c_{i}} P_{j-1}(x) \mathrm{d} x\right), \mathscr{P}_{s}=\left(P_{j-1}\left(c_{i}\right)\right) \in \mathbb{R}^{k \times s}, \quad \Omega=\operatorname{diag}(\boldsymbol{b}),
$$

where $\left\{P_{j}\right\}_{j \geq 0}$ is Legendre polynomial basis orthonormal on the interval $[0,1], P_{i} \in \Pi_{i}, \int_{0}^{1} P_{i}(x) P_{j}(x) \mathrm{d} x=\delta_{i j}, \forall i, j \geq$ 0 , having set $\Pi_{i}$ the set of polynomials of degree at most $i, \delta_{i j}$ the Kronecker symbol, and $\boldsymbol{b}=\left(b_{1}, \ldots, b_{k}\right)^{\top}$, $\boldsymbol{c}=\left(c_{1}, \ldots, c_{k}\right)^{\top}$ the vectors with the $k$-th Legendre weights and abscissae (i.e., $P_{k}\left(c_{i}\right)=0, i=1, \ldots, k$ ), respectively. A thorough analysis of such methods can be found in [9], showing that they have order $2 s$, and their efficient implementation has been considered in $[8,2]$. In particular, the so called blended implementation for solving the discrete problems generated by HBVMs, which is described in [8,3] (see also [4]), allows the use of huge values of $s$. This, in turn, permits the use of HBVMs as spectral methods in time, as it has been proposed in [5, 12], for efficiently solving highly/stiffly-oscillatory problems. A thorough analysis of spectral HBVMs (SHBVMs) (i.e., HBVMs used as spectral methods in time), has been recently devised in [1], which explains their effectiveness also in the case where the used time-step is not small (as is the case, when speaking about spectral methods). In the sequel, we report the main results of the analysis carried out in [1], and provide a few examples showing the potentialities of SHBVMs as general ODE solvers, able to provide a numerical solution with full machine accuracy.

\section{SPECTRAL HAMILTONIAN BOUNDARY VALUE METHODS (SHBVMS)}

Let us consider the numerical solution of the following ODE-IVP which, for sake of simplicity, we take in the form

$$
\dot{y}(t)=f(y(t)), \quad t \in[0, h], \quad y(0)=y_{0} \in \mathbb{R}^{m},
$$

where $h$ is the used time-step (we are indeed speaking about a one-step method) and $f$ is assumed to be, for sake of brevity, analytical. Following the approach in [9], we can expand the right-hand side of (2) along the Legendre polynomial basis $\left\{P_{j}\right\}_{j \geq 0}$ as follows:

$$
\dot{y}(c h)=\sum_{j \geq 0} P_{j}(c) \gamma_{j}(y), \quad c \in[0,1], \quad \gamma_{j}(y)=\int_{0}^{1} P_{j}(\tau) f(y(\tau h)) \mathrm{d} \tau, \quad j \geq 0 .
$$


A polynomial approximation $\sigma \in \Pi_{s}$ can then be obtained from (3) by truncating the series to a finite sum:

$$
\dot{\sigma}(c h)=\sum_{j=0}^{s-1} P_{j}(c) \gamma_{j}(\sigma), \quad \Rightarrow \quad \sigma(c h)=y_{0}+h \sum_{j=0}^{s-1} \int_{0}^{c} P_{j}(x) \mathrm{d} x \gamma_{j}(\sigma), \quad c \in[0,1],
$$

where $\gamma_{j}(\sigma)$ is formally defined as in (3) by replacing $y$ with $\sigma$. Moreover, by approximating the Fourier coefficients by the Gauss-Legendre formula of order $2 k$,

$$
\gamma_{j}(\sigma)=\int_{0}^{1} P_{j}(\tau) f(\sigma(\tau h)) \mathrm{d} \tau \approx \sum_{i=1}^{k} b_{i} P_{j}\left(c_{i}\right) f\left(\sigma\left(c_{i} h\right)\right)=: \hat{\gamma}_{j}
$$

one obtains the $\operatorname{HBVM}(k, s)$ method (1). Here, for sake of brevity we continue to use the same symbol $\sigma$ for the polynomial approximation obtained using the quadrature. The following result holds true [9].

Theorem $1 \gamma_{j}(y), \gamma_{j}(\sigma)=O\left(h^{j}\right)$. For all $k \geq s: \hat{\gamma}_{j}=O\left(h^{j}\right)$ and, moreover, setting $y_{1}=\sigma(h) \equiv y_{0}+h \gamma_{0}(\sigma)$, one has: $y_{1}-y(h)=O\left(h^{2 s+1}\right)$.

In other words, the previous result states that we are speaking about an order $2 s$ method. However, there is evidence $[5,12]$ that the method can be effectively used with large (and even huge) time-steps, provided that $s$ is large enough. Before going into details, at first we shall use, in (5), $k$ large enough so that $\hat{\gamma}_{j} \doteq \gamma_{j}(\sigma)$, where $\doteq$ means "equal within the round-off error level". As an example, for the double precision IEEE, the choice

$$
k=\max \{20, s+2\}
$$

is generally sufficient for this purpose. Assuming this holds true in the sequel, the following result summarizes the theoretical achievements in [1].

Theorem 2 Let $f(y(z))$ be analytical in a closed ball of radius $r^{*}$ centered at 0 . Then, for all $0<h \leq h^{*}<r^{*}$, there exist $\kappa=\kappa\left(h^{*}\right), \rho:=\frac{r^{*}}{h} \geq \rho^{*} \equiv r^{*} / h^{*}>1$, and $M=M\left(h^{*}\right)$ such that (see (4)), considering any suitable vector norm $|\cdot|$ :

$$
\left|\gamma_{j}(\sigma)\right| \leq \frac{\kappa}{\sqrt{j+1}} \rho^{-j}, \quad j=0,1, \ldots, s-1, \quad|\sigma(h)-y(h)| \leq h M \rho^{-2 s} .
$$

This result is interesting from many points of view. In fact, one has that, when the solution is regular enough, then one is allowed to use quite large time-steps, still getting an accurate approximation $y_{1} \equiv \sigma(h)$. Moreover, in the implementation of the methods, the coefficients $\hat{\gamma}_{j} \doteq \gamma_{j}(\sigma)$ are computed. Therefore, by virtue of (7) one can estimate the parameters $\kappa$ and $\rho$, thus getting a corresponding error estimate. As a consequence, again from (7), one can dynamically vary both $s$ and $h$, in order to gain a desired accuracy (we refer to [1] for more details). In particular, one can expect a fully accurate approximation to the solution, within the limit of the used finite precision arithmetic, by requiring $\left|\gamma_{s}(\sigma)\right| /\left|\gamma_{0}(\sigma)\right|$ to be of the order of the square root of the machine epsilon $u$. In the sequel, we report a few numerical examples, aimed at showing the potentialities of this approach, dealing with different ODE problems obtained by a Matlab implementation of the method.

A Kepler problem. We consider, at first, the following Kepler problem,

$$
\dot{q}=p, \quad \dot{p}=-q /\|q\|_{2}^{3}, \quad t \in[0,2 \pi], \quad q(0)=(0.5,0)^{\top}, \quad p(0)=(0, \sqrt{3})^{\top},
$$

whose solution is periodic of period $2 \pi$. We fix a time-step $h=2 \pi / 10$ and we show, in the plot on the left of Figure 1 , the norms of $\hat{\gamma}_{j}, j=0,1, \ldots, 29$ (triangles), obtained in the first step of integration using $k=32$ in (5)-(6), along with the estimated upper bound (7) (solid line and circles), with estimated $\kappa \approx 35.6$ and $\rho \approx 3.5$. On the right of the same figure, is the plot of the solution error at $t=2 \pi$ versus $s$, having fixed the same time-step $h=2 \pi / 10$ : as one may see, after $s=14$, a fully accurate solution is obtained. This well match the fact that $\left|\hat{\gamma}_{14}\right| /\left|\hat{\gamma}_{0}\right| \approx \sqrt{u}$. 

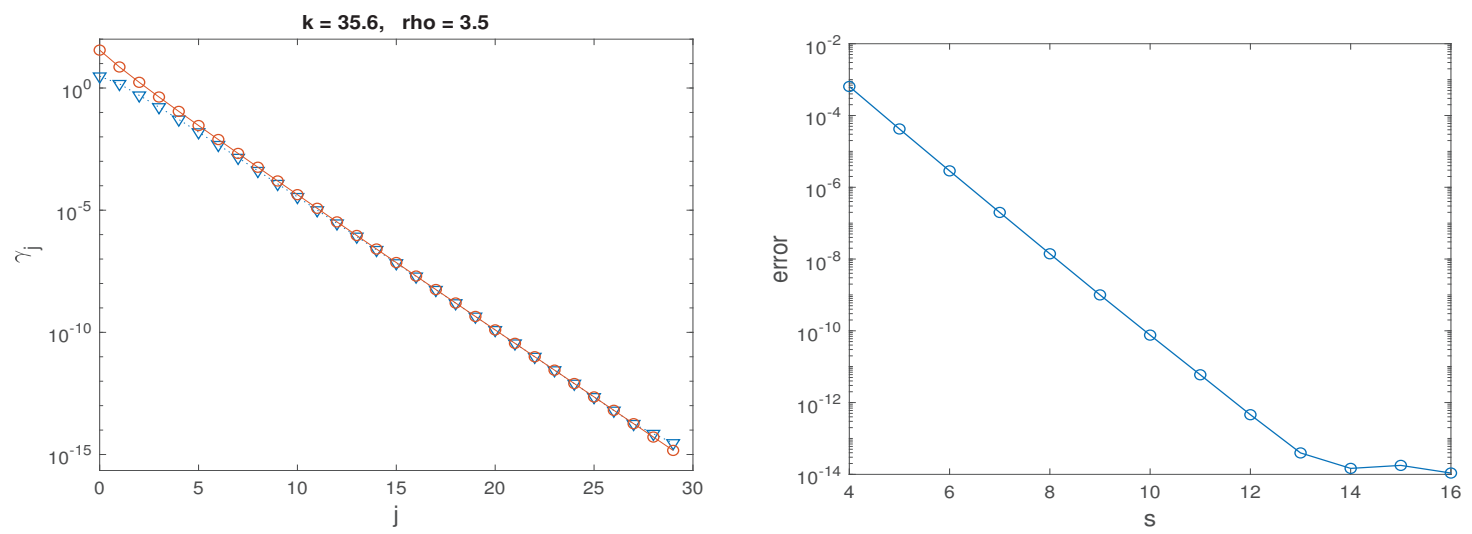

FIGURE 1. Kepler problem (8).
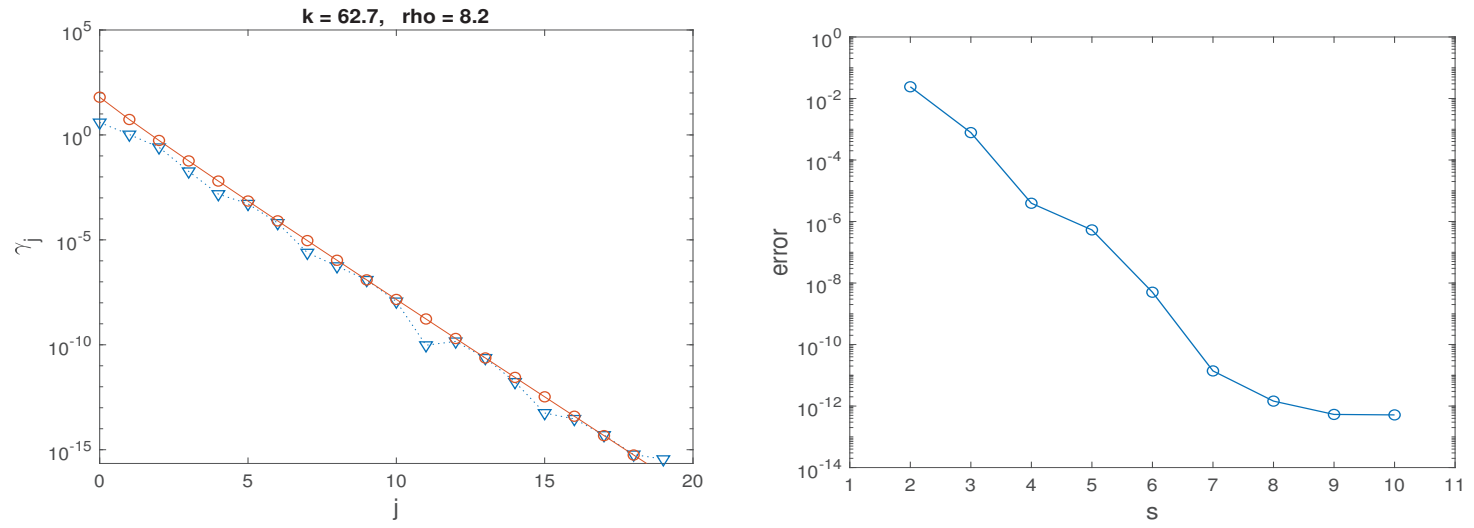

FIGURE 2. Lotka-Volterra problem (9).

A Lotka-Volterra problem. Similarly as done above, we now consider the following Lotka-Volterra problem,

$$
\dot{y}=\left(\begin{array}{ccc}
0 & -0.5 y_{1} y_{2} & 0.5 y_{1} y_{3} \\
0.5 y_{1} y_{2} & 0 & -y_{2} y_{3} \\
-0.5 y_{1} y_{3} & y_{2} y_{3} & 0
\end{array}\right)\left(\begin{array}{c}
2 \\
1+y_{2}^{-1} \\
2-2 y_{3}^{-1}
\end{array}\right), \quad t \in[0, T], \quad y(0)=\left(\begin{array}{c}
1 \\
1.9 \\
0.5
\end{array}\right)
$$

having a periodic solution of period $T \approx 2.878130103817$. Again, we fix a time-step $h=T / 10$ and we show, in the plot on the left of Figure 2, the norms of $\hat{\gamma}_{j}, j=0,1, \ldots, 19$ (triangles), obtained in the first step of integration using $k=22$ in (5)-(6), along with the estimated upper bound (7) (solid line and circles), with estimated $\kappa \approx 62.7$ and $\rho \approx 8.2$. On the right of the same figure, is the plot of the solution error at $t=T$ versus $s$, having fixed the same time-step $h=T / 10$ : as one may see, after $s=9$, a fully accurate solution is obtained. This well match the fact that, also in this case, $\left|\hat{\gamma}_{9}\right| /\left|\hat{\gamma}_{0}\right| \approx \sqrt{u}$.

A stiff problem. At last, let us now consider the following stiff problem,

$$
\dot{y}=\left(\begin{array}{ccc}
-9999 & 1 & 1 \\
9900 & -100 & 1 \\
98 & 98 & -2
\end{array}\right)(y-g(t))+\dot{g}(t), \quad t \in[0,10], \quad y(0)=g(0), \quad g(t)=\left(\begin{array}{c}
\cos 2 \pi t \\
\cos 4 \pi t \\
\cos 6 \pi t
\end{array}\right),
$$

whose solution is $y(t)=g(t)$. We now fix a time-step $h=1$ and report, in the plot on the left of Figure 3 , the norms of $\hat{\gamma}_{j}, j=0,1, \ldots, 39$ (triangles), obtained in the first step of integration using $k=42$ in (5)-(6), along with the estimated upper bound (7) (solid line and circles), with estimated $\kappa \approx 3 \cdot 10^{12}$ and $\rho \approx 5.8$. On the right of the same figure, is the plot of the solution error at $t=10$ versus $s$, having fixed the same time-step $h=1$ : as one may see, after $s=25$, a fully accurate solution is obtained. This well match the fact that, also in this case, $\left|\hat{\gamma}_{25}\right| /\left|\hat{\gamma}_{0}\right| \approx \sqrt{u}$. 

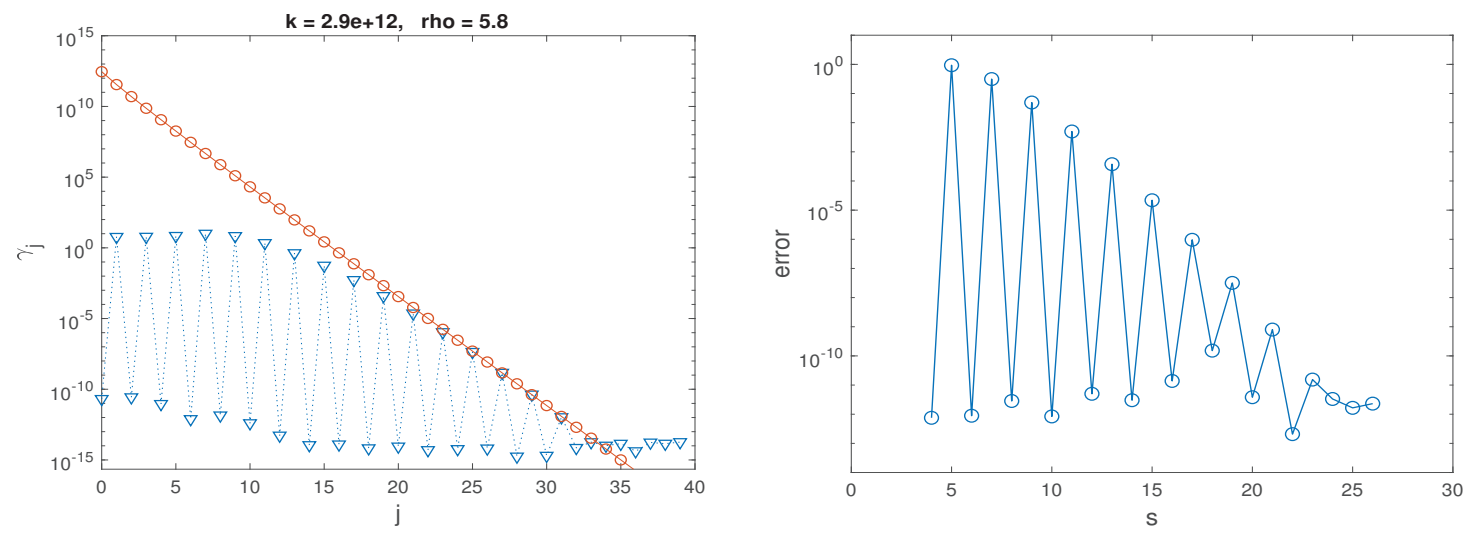

FIGURE 3. Stiff problem (10).

Conclusions. In this paper we have provided some numerical tests confirming the potentialities of obtaining an efficient, general purpose ODE solver, able to get numerical solutions with full machine accuracy. As a motivation for requiring full accuracy is that possible constants of motion inherent to the dynamical system would be precisely conserved by the integrator modulo the underlying machine epsilon $[5,1]$. The solver relies on the use of Hamiltonian Boundary Value Methods as spectral methods in time. Last but not least, this use of the methods is made possible by the availability of the very efficient blended iteration for solving the generated discrete problems. This latter nonlinear iteration, devised in [8] for HBVMs, dates back to previous work on block implicit methods (see, e.g., [10, 11] and references therein). It is worth mentioning that, due to the fast convergence properties of the blended iteration, the overall execution times are, in most interesting cases, definitely more favourable when the spectral implementation of the methods is considered.

\section{REFERENCES}

1. P. Amodio, L. Brugnano, F. Iavernaro. Analysis of Spectral Hamiltonian Boundary Value Methods (SHBVMs) for the numerical solution of ODE problems. Numer. Algorithms (2019) https: / / doi.org/10.1007/s11075-019-00733-7

2. L. Brugnano, G. Frasca Caccia, F. Iavernaro. Efficient implementation of Gauss collocation and Hamiltonian Boundary Value Methods. Numer. Algorithms 65, 633-650 (2014).

3. L. Brugnano, F. Iavernaro. Line Integral Methods for Conservative Problems. Chapman and Hall/CRC, Boca Raton, FL, 2016.

4. L. Brugnano, F. Iavernaro. Line Integral Solution of Differential Problems. Axioms 7(2), 36 (2018) https : / / do i . org/ $10.3390 /$ axioms 7020036 .

5. L. Brugnano, F. Iavernaro, J.I. Montijano, L. Rández. Spectrally accurate space-time solution of Hamiltonian PDEs. Numer. Algorithms (2018) http://doi.org/10.1007/s11075-018-0586-z

6. L. Brugnano, F. Iavernaro, D. Trigiante. Hamiltonian BVMs (HBVMs): A family of "drift free" methods for integrating polynomial hamiltonian problems. AIP Conference Proc. 1168, 715-718 (2009).

7. L. Brugnano, F. Iavernaro, D. Trigiante. Hamiltonian Boundary Value Methods (Energy Preserving Discrete Line Integral Methods). JNAIAM J. Numer. Anal. Ind. Appl. Math. 5, 17-37 (2010).

8. L. Brugnano, F. Iavernaro, D. Trigiante. A note on the efficient implementation of Hamiltonian BVMs. J. Comput. Appl. Math. 236, 375-383 (2011).

9. L. Brugnano, F. Iavernaro, D. Trigiante. A simple framework for the derivation and analysis of effective one-step methods for ODEs. Appl. Math. Comput. 218, 8475-8485 (2012).

10. L. Brugnano, C. Magherini. Blended Implementation of Block Implicit Methods for ODEs. Appl. Numer. Math. 42, 29-45 (2002).

11. L. Brugnano, C. Magherini. Recent Advances in Linear Analysis of Convergence for Splittings for Solving ODE problems. Appl. Numer. Math. 59, 542-557 (2009).

12. L. Brugnano, J.I. Montijano, L. Rández. On the effectiveness of spectral methods for the numerical solution of multifrequency highly-oscillatory Hamiltonian problems. Numer. Algorithms (2018) http://dx.doi.org/10.1007/ s11075-018-0552-9 